\title{
Growth of Finitely Presented Rees Quotients of Free Inverse Semigroups
}

\author{
Dedicated to Professor John Meakin
}

\section{L.M. ShneErson and D. EAsdown}

\begin{abstract}
We prove that a finitely presented Rees quotient of a free inverse semigroup has polynomial growth if and only if it has bounded height. This occurs if and only if the set of nonzero reduced words has bounded Shirshov height and all nonzero reduced but not cyclically reduced words are nilpotent. This occurs also if and only if the set of nonzero geodesic words have bounded Shirshov height. We also give a simple sufficient graphical condition for polynomial growth, which is necessary when all zero relators are reduced. As a final application of our results, we give an inverse semigroup analogue of a classical result that characterises polynomial growth of finitely presented Rees quotients of free semigroups in terms of primitive words that label loops of the Ufnarovsky graph of the presentation.
\end{abstract}

\section{INTRODUCTION}

In [21] the authors initiated the study of growth of finitely presented Rees quotients of free inverse semigroups and the relationship with satisfiability of identities. In [5] they considered satisfiability of identities in signature with involution in the one relator case. Lau considered rationality of growth series [9] and degrees of growth [10] for semigroups from this class (see also [20] by the first author, in the wider context of Gelfand-Kirillov dimension and Rees quotients using infinitely many relators). In [21], growth was shown to be polynomial or exponential for semigroups from this class and an algorithmic criterion given to recognise which type of growth occurs [21, Section 3]. The arguments relied on a graphical technique, the ingredients of which are outlined in the next section, which is a modification of an idea due to Ufnarovsky [23] [24] (see also [13, Chapter 24]). In Theorem 3.3 below a simplified graphical condition is given which is always sufficient for polynomial growth, and necessary when all zero relators are reduced. Shirshov [18] (see also [16, Chapter 4], [22]) introduced the notion of height in his celebrated study of rings which satisfy a polynomial identity. In Theorem 4.3 in the final section we give simple criteria for polynomial growth for semigroups from our class in terms of height, nilpotency and geodesic words. Some of the main results of the final section also appear in Chapter 4 of Lau's doctoral thesis [11], but with different proofs. The final result, Theorem 4.4, is an application of our results to yield an inverse semigroup analogue of a classical result characterising polynomial growth 
of finitely presented Rees quotients of free semigroups in terms of primitive words that label loops of the Ufnarovsky graph.

\section{Preliminaries}

We assume familiarity with the basic definitions and elementary results from the theory of semigroups, which can be found in any of [3], [7], [8] or [14]. Let $S$ be a semigroup generated by a finite subset $X$. Recall that the length $l(t)$ of an element $t \in S$ (with respect to $X$ ) is the least number of factors in all representations of $t$ as a product of elements of $X$, and

$$
g_{S}(m)=|\{t \in S \mid l(t) \leq m\}|
$$

is called the growth function of $S$. We say that $S$ has polynomial growth if there exist natural numbers $q$ and $d$ such that $g_{S}(m) \leq q m^{d}$ for all natural numbers $m$, and exponential growth if there exists a real number $\alpha>1$ such that $g_{S}(m) \geq \alpha^{m}$ for all sufficiently large $m$. These definitions and notions of growth apply also to subsets of $S$ in an obvious way. Throughout this paper, we may implicitly assume that zero lies in the generating set of any semigroup with zero (so that zero always has length one). If $t \in S$ and the equation $t=w$ holds in $S$, where $w$ is a product of $\ell(t)$ elements of $X$ regarded as a word over the alphabet $X$, then we call the word $w$ a geodesic for $t$ (with respect to $X$ ).

Let $A$ be a finite alphabet and put $B=A \cup A^{-1}$, where it is understood that the union is disjoint and any $a$ in $A$ may also be denoted by $\left(a^{-1}\right)^{-1}$. The free semigroup and free inverse semigroup over $A$ are denoted by $F_{A}$ and $F I_{A}$ respectively. Literal equality of words is denoted by the symbol $\overline{\overline{ }}$, and $|w|$ denotes the number of letters in a word $w$. Recall that $w$ is reduced if $w$ does not contain $x x^{-1}$ as a subword for any letter $x \in B$, and that $w$ is cyclically reduced if $w$ and $w^{2}$ are both reduced (whence all powers of $w$ are reduced). A word is called primitive if it cannot be expressed as a power of a proper subword. Recall that elements of $F I_{A}$ may be regarded as birooted word trees (introduced in [12]), the terminology and theory of which are explained in [2] (see also [21, Section 2]). As in [21], denote the word tree of a word $w$ over $B$ by $T(w)$. If $u$ and $v$ are elements of $F I_{A}$, then $T(u)$ is a subtree of $T(v)$ if and only if $v$ may be expressed as a product of elements, one of whose factors is $u$, in which case we say that $u$ divides $v$. Any reference to Green's relation $\mathcal{J}$ will be with repect to $F I_{A}$. Note that two words over $A \cup A^{-1}$ are $\mathcal{J}$-related if and only if their word trees are identical. Recall that an element $s$ of a semigroup $S$ with zero is nilpotent if some power of $s$ is zero.

Any given element $w$ of $F I_{A}$ may also be expressed as

$$
w=u_{1} u_{1}^{-1} u_{2} u_{2}^{-1} \ldots u_{r} u_{r}^{-1} \bar{w}
$$


for some nonnegative integer $r$ and reduced words $u_{1}, \ldots, u_{r}, \bar{w}$. If $r$ is as small as possible, so that no $u_{i}$ can be an initial segment of $u_{j}$ for $i \neq j$, then (as in [20]) we call (1) the Schein (left) canonical form of $w$, which is unique up to order of idempotents. Note that $r$ is bounded by the number of leaf vertices on $T(w)$. We refer to the idempotents $u_{1} u_{1}^{-1}, \ldots, u_{r} u_{r}^{-1}$ as the components and $\bar{w}$ as the irreducible part of $w$.

Let $w$ be a nonempty word over $B$. A geodesic $\widetilde{w}$ for $w$ in $F I_{A}$ has the form

$$
\widetilde{w} \underline{\overline{0}} u_{0} e_{1} u_{1} e_{2} \ldots e_{n} u_{n}
$$

where $n \geq 0$ is the number of idempotents in the Schein canonical form,

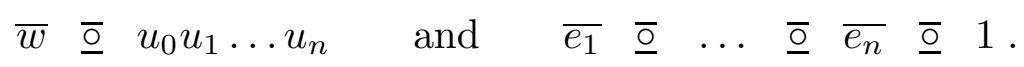

Geodesics in free inverse monoids were considered by Choffrut [4, Section 3.2], and also by Poliakova and Schein [15], where they gave a rewriting system that reduces an arbitrary word in the free inverse semigroup to its geodesic form (which is unique up to the representation of idempotents). To form each word $e_{i}$ representing an idempotent, one systematically traces the labels of edges of the branches of the word tree that emanate from the geodesic chain of $T(w)$ labelled by $\bar{w}$ at the vertex pointed to by the last letter of $u_{i-1}$ (or the initial vertex if $i=1$ and $u_{0}$ is empty). The word $e_{i}$ is not unique unless there is exactly one branch emanating from that vertex and that branch is a chain, because one may arbitrarily choose the order of multiple subbranches to trace from any given vertex. The number of occurrences of a given letter in $e_{i}$ however is unique.

As in [21], let $\mathfrak{M}_{F I}$ denote the class of finitely presented inverse semigroups $S$ with zero having a presentation of the form

$$
\left.S=\langle A| c_{i}=0 \quad \text { for } \quad i=1, \ldots, k\right\rangle
$$

where $A$ is some finite alphabet, $k$ some nonnegative integer and $c_{i} \in F_{A \cup A^{-1}}$ for $i=1$ to $k$. Then $\mathfrak{M}_{F I}$ is precisely the class of Rees quotients of finitely generated free inverse semigroups by finitely generated ideals. We refer to the words $c_{1}, \ldots, c_{k}$ appearing in the above presentation as (zero) relators. Note that $S$ is unaffected by replacing any relator by a word to which it is $\mathcal{J}$-equivalent.

We now redefine the Ufnarovsky graph $\Gamma=\Gamma_{S}$ of $S$ (depending on the presentation of $S$ ). The illustrative example given below in Section 3 may assist the reader in digesting the following construction. Suppose that there is at least one relator which is $\mathcal{J}$-equivalent to reduced word of length $\geq 2$. (This is a very mild restriction for our purposes, and cases when it does not hold are catalogued in comments following the statement of Theorem 2.1.) Note that the definition of vertices given below is slightly different to that given in [21, Section 3]. This modification shortens calculations when long relators appear which are not $\mathcal{J}$ equivalent to reduced words. Put $d+1=\max \left\{l\left(c_{i}\right) \mid i=1, \ldots, k\right\}$ and

$$
\bar{d}+1=\max \{l(c) \mid c \text { is a reduced word } \mathcal{J} \text {-equivalent to some relator }\} .
$$


Note that $\bar{d}$ may be calculated easily by inspecting word trees of relators. Also $1 \leq \bar{d} \leq d$. Vertices of $\Gamma$ are defined to be reduced words of length $\bar{d}$ which are nonzero in $S$. If $v_{1}$ and $v_{2}$ are vertices then a directed edge from $v_{1}$ to $v_{2}$ is defined in $\Gamma$ if there exist letters $g, h \in A \cup A^{-1}$ such that $v_{1} g$ is a reduced word which is nonzero in $S$ and $v_{1} g$ 으 $h v_{2}$. We regard the letter $g$ as a label for this edge. Paths in $\Gamma$ may then be labelled by reduced words which are nonzero in $S$. Conversely if $w$ 의 $v u \underline{\overline{ }} u^{\prime} v^{\prime}$ is any nonzero reduced word where $v$ and $v^{\prime}$ have length $\bar{d}$ then $u$ labels a path in $\Gamma$ emanating from $v$ and terminating at $v^{\prime}$.

By a cycle in $\Gamma$ we mean a path which starts and finishes at the same vertex. By a loop at a vertex $v$ we mean a cycle which begins at $v$ using no other vertex more than once. Recall from [21, Section 3] that $(z, P)$ is an adjacent pair if $z$ is a reduced word which labels a loop in $\Gamma$ at a vertex $v$ and $P$ is a letter labelling an edge which emanates from $v$ and terminates outside the loop. The proof of [21, Theorem 1] requires only trivial modifications to yield

TheOREM 2.1. Let $S=\langle A| c_{i}=0$ for $\left.i=1, \ldots, k\right\rangle$ be an inverse semigroup from the class $\mathfrak{M}_{F I}$ and suppose that $\bar{d} \geq 1$. Then the following conditions are equivalent:

(a) $S$ has polynomial growth.

(b) $S$ does not contain any noncyclic free subsemigroup.

(c) (i) $\Gamma_{S}$ has no vertex contained in different cycles; and

(ii) if $(z, P)$ is an adjacent pair in $\Gamma_{S}$ then

$$
z^{d+1} P P^{-1} z^{d+1}=0 \quad \text { in } S .
$$

Note that if $\bar{d}=0$ then relators that are $\mathcal{J}$-related to reduced words are $\mathcal{J}$ related to generators, in which case such generators and any relators in which they or their inverses occur may be deleted without altering $S$ (up to isomorphism). If $\bar{d}$ does not exist then no relator is $\mathcal{J}$-related to a reduced word, so that the word tree of any relator must have at least one vertex of degree $>2$, in which case either (i) $A=\{a\}$ and there are no relators, so that $S$ is a free monogenic inverse semigroup with zero, which has polynomial (in fact cubic) growth, or (ii) $A$ contains distinct letters $a$ and $b$ that generate a nonmonogenic free subsemigroup of $S$, so that the growth of $S$ is exponential. These observations and the previous theorem tell us (as in [21, Corollaries 1 and 2]) that any Rees quotient of a finitely generated free inverse semigroup by a finitely generated ideal has polynomial or exponential growth (and there is an algorithm to determine which type of growth occurs).

The proof of [21, Theorem 1] relied on a delicate argument that bounds the number of idempotents in the Schein canonical form when the semigroup $S$ has polynomial growth. From that proof we extract the following result for use in the final section of this paper: 
Scholium 2.2. Let $S$ be an inverse semigroup from the class $\mathfrak{M}_{F I}$ having polynomial growth where $\bar{d} \geq 1$. Form the Ufnarovsky graph $\Gamma_{S}$ and let $N$ be the number of paths in $\Gamma_{S}$ in which no cycle is repeated, and $T$ the maximum number of cycles in any such path. Put

$$
\lambda=(2 d+2)^{T}+1 \quad \text { and } \quad M=\lambda N+(d-1)(2|A|)^{d-1} .
$$

Then any nonzero element of $S$ has at most $M$ components in its Schein canonical form. In particular, the number of leaf vertices of $T(w)$ for any word $w$ that is nonzero in $S$ is bounded by $M+2$.

Finally we recall the notion of height introduced by Shirshov [18]. Let $S$ be any semigroup and $X$ a subset of $S$. Denote by $(X)$ the subsemigroup of $S$ generated by $X$. If $s \in(X)$ can be expressed as a product

$$
s=h_{1}^{\alpha_{1}} \ldots h_{k}^{\alpha_{k}}
$$

for some $h_{1}, \ldots, h_{k} \in X$ and positive integers $\alpha_{1}, \ldots, \alpha_{k}$, and $k$ is as small as possible, then we say the height of $s$ with respect to $X$ is $k$. We say that a subset $T$ of $S$ has bounded height $\leq k$ if there exists a finite subset $X$ of $S$ such that $T \subseteq(X)$ and the height of elements of $T$ with respect to $X$ is at most $k$. In the final section of this paper we prove that, for semigroups from the class $\mathfrak{M}_{F I}$, the properties of having polynomial growth and having bounded height are equivalent.

Semigroups with bounded height in general need not have polynomial growth (for example, the Baumslag-Solitar groups $B S(1, k)$ for $|k|>1[2]$ ), and so various authors have placed restrictions that guarantee polynomial growth (see, for example, $[20,1,25])$. For example, suppose in addition to the above there is an ambient generating set $Y$ for $S$ (so for semigroups from the class $\mathfrak{M}_{F I}$ we would take $Y$ to be $B$ ). If $k$ can always be chosen minimally in (2) so that the word $h_{1}^{\alpha_{1}} \ldots h_{k}^{\alpha_{k}}$ is a geodesic for $s$ (with respect to $Y$ ) then we call $k$ the Shirshov height of $s$. We say a subset $T$ of $S$ has bounded Shirshov height $\leq k$ if each element of $T$ has Shirshov height and this is at most $k$. For example, in the simplest nontrivial case, if $S$ is the free monogenic inverse semigroup generated by a then its subset of nonzero reduced words has bounded height 1 (being the set of positive and negative powers of $a$ ), and $S$ has bounded Shirshov height 3 using geodesic forms.

It is very easy to see that if $S$ has bounded Shirshov height $k$ then $S$ has polynomial growth (with respect to the ambient generating set $Y$ ) of degree at most $k$. In all the cases considered below in this paper where we establish bounded Shirshov height, if $s$ is also geodesic in (2) then the number of occurrences of any given letter in $B$ is the same on both sides of (2). Thus, for the cases we consider, our notion in fact coincides with the original classical notion [18].

The following facts are used in the final section. The first is routine and wellknown. For the second, the following notation is convenient: denote by $\|w\|$ the number of vertices of $T(w)$ where $w$ is any word over $B$. 
Lemma 2.3. If $T$ is a subset of a free semigroup $F_{A}$ where $A$ is a finite alphabet and $T$ has bounded height, then $T$ has polynomial growth.

Lemma 2.4 Suppose that $T$ is a set of words over $B=A \cup A^{-1}$ where $A$ is a finite alphabet. If $T$ has bounded height in the free inverse semigroup $F I_{A}$ then $T$ has polynomial growth in $F I_{A}$. In particular, if a Rees quotient of a finitely generated free inverse semigroup has bounded height then it has polynomial growth.

Proof. Suppose that $T$ has height $\leq K$ with respect to some finite set $X$ of words over $B$. Consider $w \in T$. Then $w=v$ in $F I_{A}$ where

$$
v \quad \underline{\overline{ }} \quad h_{1}^{\alpha_{1}} \ldots h_{k}^{\alpha_{k}}
$$

for some $k \leq K$, words $h_{1}, \ldots, h_{k} \in X$ and positive integers $\alpha_{1}, \ldots, \alpha_{k}$. If $h_{i}$ is not idempotent then the cyclically reduced part of $\overline{h_{i}}$ is nonempty, and it follows quickly that $\alpha_{i}<\left\|h_{i}^{\alpha_{i}}\right\|$. If $h_{i}$ is idempotent then we may assume $\alpha_{i}=1$. Observe that $h_{i}^{\alpha_{i}}$ divides $w$ so that in all cases

$$
\alpha_{i} \leq\left\|h_{i}^{\alpha_{i}}\right\|-1 \leq\|w\|-1 \leq|\widetilde{w}|=\ell(w)
$$

where $\ell(w)$ is calculated in $F I_{A}$ with respect to $B$. This produces the constraint

$$
\alpha_{1}+\ldots+\alpha_{k} \leq K \ell(w)
$$

from which it follows readily that the growth of $T$ in $F I_{A}$ with respect to $B$ is bounded above by some polynomial of degree $\leq K$, completing the proof of the lemma.

\section{Growth}

In this section we give a useful sufficient condition for polynomial growth in terms of the Ufnarovsky graph. This condition is also necessary when all the relators are reduced. We begin with two observations concerning the existence of loops and adjacent pairs. We assume for the time being that $S$ has a presentation of the form given in the previous section and $\bar{d} \geq 1$.

Lemma 3.1. Any word labelling a loop in $\Gamma_{S}$ is primitive.

Proof. Suppose that $z^{\alpha}$ labels a loop emanating from a vertex $v$. Then $z^{\alpha \bar{d}}$ labels a cycle ending at $v$, so $v$ must be a suffix of $z^{\alpha \bar{d}}$. If $v \underline{\overline{\mathrm{O}}} u z^{\beta}$ for some $\beta \geq 1$ and words $u, u^{\prime}$ such that $z$ 으 $u^{\prime} u$ then $v z \underline{\overline{0}} u u^{\prime} v$. If $z \overline{\overline{ }} v^{\prime} v$ for some $v^{\prime}$ then $v z \underline{\overline{ }} v v^{\prime} v$. In both cases $z$ labels a path beginning and ending at $v$. Hence, since $z^{\alpha}$ labels a loop, $\alpha=1$, completing the proof. 
Lemma 3.2. Suppose that $\Gamma_{S}$ contains no vertex in different cycles, that $v$ is a nonempty word, $P$ is a letter that is not the first letter of $v$, and $v^{d+1} P$ is reduced and nonzero in $S$. Then $v \underline{\overline{\mathrm{o}}} z^{\alpha}$ for some word $z$ and positive integer $\alpha$ such that $(z, P)$ is an adjacent pair in $\Gamma_{S}$.

Proof. Certainly $v^{d+1}$ is nonzero in $S$. This implies that all divisors of powers of $v$ are nonzero in $S$, since all relators have length $\leq d+1$. Write $v^{\bar{d}} \underline{\overline{0}} v_{1} v_{2}$ where $v_{2}$ has length $\bar{d}$. Then $v_{2} v^{\bar{d}+1} \underline{\overline{\mathrm{o}}} v_{2} v v^{\bar{d}} \underline{\overline{\mathrm{o}}}\left(v_{2} v v_{1}\right) v_{2}$. But $v_{2} v^{\bar{d}+1}$ is reduced and nonzero in $S$, since it divides a power of $v$. Hence $v^{\bar{d}+1}$ labels a cycle beginning and ending at $v_{2}$. Let $z$ be the smallest suffix of $v^{\bar{d}+1}$ which labels a loop at $v_{2}$. Since $\Gamma_{S}$ contains no vertex in different cycles, $v^{\bar{d}+1}$ is a positive power of $z$. Hence both $v$ and $z$ must be positive powers of some word $w$. By Lemma 3.1, z $\overline{\mathrm{c}} w$. Further, since $v^{\bar{d}+1} P$ is reduced and nonzero in $S$ and again since no vertex is contained in different cycles, $P$ must label an edge emanating from $v_{2}$ which terminates outside the loop labelled by $z$. Hence $(z, P)$ is an adjacent pair in $\Gamma_{S}$ and the lemma is proved.

Theorem 3.3. Let $S=\langle A| c_{i}=0$ for $\left.i=1, \ldots, k\right\rangle$ be an inverse semigroup from the class $\mathfrak{M}_{F I}$ where $\bar{d} \geq 1$. Then $S$ has polynomial growth if

(i) $\Gamma_{S}$ has no vertex contained in different cycles; and

(ii) if $(z, P)$ is any adjacent pair then $\left(z^{-1}, P\right)$ is not an adjacent pair.

If $S$ has polynomial growth and each relator is $\mathcal{J}$-equivalent to a reduced word, then (i) and (ii) hold.

Proof. Suppose that conditions (i) and (ii) hold and that $(z, P)$ is an adjacent pair. Suppose that $z^{d+1} P P^{-1} z^{d+1}$ is nonzero in $S$. Then $\left(z^{-1}\right)^{d+1} P$ is reduced and nonzero in $S$. Hence by Lemma 3.2, $z^{-1} \underline{\overline{0}} w^{\alpha}$ for some word $w$ and positive integer $\alpha$ such that $(w, P)$ is an adjacent pair. But $z^{-1}$ labels a loop in $\Gamma_{S}$, since $z$ labels a loop and inversion induces an edge-reversing bijection of the graph. Hence, by Lemma 3.1, $\alpha=0$. Thus $\left(z^{-1}, P\right)$ is an adjacent pair, contradicting (ii). Hence $z^{d+1} P P^{-1} z^{d+1}=0$ in $S$. By Theorem 2.1, $S$ has polynomial growth.

Suppose now that $S$ has polynomial growth and all relators are $\mathcal{J}$-equivalent to reduced words. Certainly (i) holds by Theorem 2.1. Let $(z, P)$ be an adjacent pair. By Theorem 2.1, $z^{d+1} P P^{-1} z^{d+1}=0$ in $S$. Hence some relator $c_{i}$ divides $z^{d+1} P P^{-1} z^{d+1}$. Since $c_{i}$ is $\mathcal{J}$-equivalent to a reduced word, its word tree is a chain of $\leq d+1$ edges, and so, by inspection, must be a subtree of the word tree of $z^{d+1} P$ or $P^{-1} z^{d+1}$. But $z^{d+1} P \neq 0$ in $S$, since $(z, P)$ is an adjacent pair. Hence $P^{-1} z^{d+1}=0$ in $S$, so $\left(z^{-1}, P\right)$ cannot be an adjacent pair. This shows that (ii) holds, and the proof of the theorem is complete. 
The condition given in the previous theorem is not in general necessary for polynomial growth. For example, consider the semigroup

$$
S=\left\langle a, b \mid a b=b^{-1} a=a b^{-1} b a=0\right\rangle .
$$

Then $\bar{d}=1, d=3$ and $\Gamma_{S}$ is the following graph:

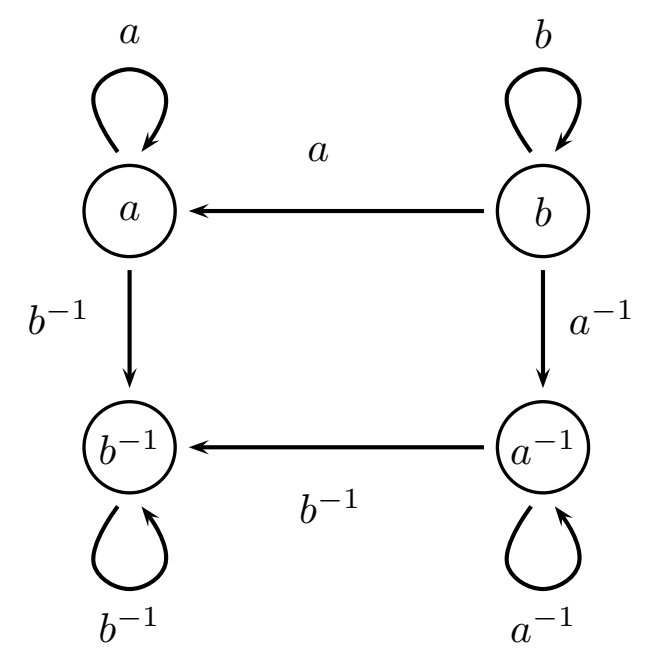

The adjacent pairs are $\left(a, b^{-1}\right),\left(a^{-1}, b^{-1}\right),\left(b, a^{-1}\right)$ and $(b, a)$, and

$$
a^{4} b^{-1} b a^{4}=a^{-4} b^{-1} b a^{-4}=b^{4} a^{-1} a b^{4}=b^{4} a a^{-1} b^{4}=0
$$

in $S$. There are no vertices in different cycles, so $S$ has polynomial growth by Theorem 2.1. However, both $\left(a, b^{-1}\right)$ and $\left(a^{-1}, b^{-1}\right)$ are adjacent pairs of $\Gamma_{S}$, so that (ii) of Theorem 3.3 fails.

\section{Bounded Height}

This section begins with a lemma that reformulates condition (c)(ii) of Theorem 2.1 in terms of nilpotent elements. The paper culminates in several equivalences relating polynomial growth to height, the main result being that the growth of a finitely presented Rees quotient of a free inverse semigroup is polynomial if and only if this quotient has bounded height.

LEMma 4.1. Let $S$ belong to the class $\mathfrak{M}_{F I}$ and suppose that no vertex of $\Gamma_{S}$ is contained in different cycles. Then $z^{d+1} P P^{-1} z^{d+1}=0$ in $S$ for all adjacent pairs $(z, P)$ in $\Gamma_{S}$ if and only if all reduced words that are not cyclically reduced are nilpotent in $S$.

Proof. Suppose that $w \underline{\overline{ }} u^{-1} v u$ is reduced, $u$ is nonempty, $v$ is cyclically reduced and $w$ is not nilpotent in $S$. Let $P$ be the first letter of $u$. Since $w^{d+1}$ 
is nonzero in $S$, and since $v^{d+1} P$ is a reduced word dividing $w^{d+1}, v^{d+1} P$ is nonzero in $S$. Hence, by Lemma 3.2, $v \underline{\mathrm{o}} z^{\alpha}$ for some word $z$ and positive integer $\alpha$ such that $(z, P)$ is an adjacent pair. But then $z^{d+1} P P^{-1} z^{d+1}$ is nonzero in $S$ since it divides $w^{2 d+2}$ and $w$ is not nilpotent.

Conversely let $(z, P)$ be an adjacent pair such that $z^{d+1} P P^{-1} z^{d+1}$ is nonzero in $S$. Put $w$ ㅇ $P^{-1} z^{d+1} P$. Observe that $w$ is a reduced but not cyclically reduced word. If $w$ is nilpotent then, by inspection, because the length of each relator is $\leq d+1$ some relator must divide $z^{d+1} P P^{-1} z^{d+1}$, a contradiction. Hence $w$ is nilpotent and the proof of the lemma is complete.

The if part of the following theorem is a standard height argument, but is included for completeness and the convenience of the reader. The only if part relies on Lemma 2.4. The result also appears as Theorem 4.3.4 of [9] with a different proof.

Theorem 4.2. Let $S$ belong to the class $\mathfrak{M}_{F I}$ and let $T$ be the set of reduced words that are nonzero in $S$. Suppose also that $\bar{d} \geq 1$. Then $T$ has bounded height [bounded Shirshov height with respect to a finite set of reduced words over $B]$ if and only if no vertex of $\Gamma_{S}$ is contained in different cycles.

Proof. Suppose firstly that no vertex of $\Gamma_{S}$ is contained in different cycles. Let $Y$ be the set of labels of loops in $\Gamma_{S}$ and put $X=Y \cup A \cup A^{-1}$, which is clearly finite. Let $l_{1}$ be the number of edges in the longest path in $\Gamma_{S}$ which contains no loops and let $l_{2}$ be the number of loops in $\Gamma_{S}$. Put $k=\bar{d}+l_{1}+l_{2}$. Let $w$ be a reduced word which is nonzero in $S$. If $l(w) \leq \bar{d}$ then certainly $w$ is a product of $\leq k$ elements of $X$. Suppose that $l(w)>\bar{d}$. Then $w$ ㅇ $v w^{\prime}$ where $v$ is a vertex of $\Gamma_{S}$ and $w^{\prime}$ is the label of a path $\pi$ in $\Gamma_{S}$ emanating from $v$. Suppose that $\pi$ has $m$ loops. No vertex of $\pi$ is contained in two different loops. Hence

$$
w \text { 으 } v P_{1}\left(x_{1} y_{1}\right)^{\alpha_{1}} x_{1} P_{2}\left(x_{2} y_{2}\right)^{\alpha_{2}} x_{2} \ldots P_{m}\left(x_{m} y_{m}\right)^{\alpha_{m}} x_{m} P_{m+1}
$$

where, for $i=1, \ldots, m, x_{i}$ labels the portion of the $i$ th loop from the vertex where $\pi$ enters to the vertex where $\pi$ exits, $y_{i}$ labels the remainder of the $i$ th loop (so that $x_{i} y_{i}$ labels the entire $i$ th loop at the vertex of entry), $P_{1}$ and $P_{m+1}$ are labels of the portions of $\pi$ which lead up to the first and away from the last loop respectively, and for $i=2, \ldots, m, P_{i}$ labels the portion of $\pi$ connecting the $(i-1)$ th to the $i$ th loop. It is understood here that any of $P_{1}, P_{m+1}, y_{1}, \ldots, y_{m}$ could be empty, but certainly all of $P_{2}, \ldots, P_{m}, x_{1}, \ldots x_{m}$ are nonempty. Observe that $l\left(P_{1} x_{1} P_{2} x_{2} \ldots P_{m} x_{m} P_{m+1}\right) \leq l_{1}$ and $m \leq l_{2}$, so that $w$ is a product of $\leq k$ powers of elements of $X$. Because we have graphical equality above of reduced words, the right hand side is automatically a geodesic for the left hand side, and this shows that $T$ has bounded Shirshov height $\leq k$ with respect to a finite set of reduced words over $B$. 
Conversely, if $\Gamma_{S}$ has a vertex in two different cycles, then the growth of $S$ is exponential, so that $T$ does not have bounded height, by Lemma 2.4, and the proof of the theorem is complete.

TheOrem 4.3. Let $S$ belong to the class $\mathfrak{M}_{F I}$. The following conditions are equivalent:

(a) S has polynomial growth.

(b) The set of reduced words that are nonzero in $S$ has bounded height [bounded Shirshov height with respect to some finite set of reduced words over B] and all reduced words that are not cyclically reduced are nilpotent.

(c) The set of geodesic words that are nonzero in $S$ has bounded Shirshov height with respect to some finite set of reduced words over $B$.

(d) $S$ has bounded height.

Proof. The equivalence of (a), (b), (c) and (d) is immediate if $\bar{d}=0$ or $\bar{d}$ does not exist, by the observations following Theorem 2.1 and by Lemma 2.3. We may suppose therefore that $\bar{d} \geq 1$.

(a) $\Longleftrightarrow(\mathrm{b})$ : This follows by Theorem 2.1, Lemma 4.1 and Theorem 4.2.

(a) $\Longrightarrow($ d): Suppose $S$ has polynomial growth. Let $T$ denote the set of reduced words that are nonzero in $S$. Because (a) implies (b), there exists a finite set $X$ such that $T$ has bounded height $k$ with respect to $X$. Using the Schein canonical form and the bound $M$ in Scholium 2.2, it is immediate then that $S$ has height bounded by $(2 M+1) k$ with respect to $X$. Thus (d) holds.

$(\mathrm{d}) \Longrightarrow(\mathrm{a})$ : This follows by Lemma 2.4.

(a) $\Longleftrightarrow(\mathrm{c})$ : As we noted it is easy to see that bounded Shirshov height implies polynomial growth (or alternatively, it is obvious that (c) implies (d), and we have just observed that (d) implies (a)). Suppose then $S$ has polynomial growth, and as before let $T$ denote the set of reduced words that are nonzero in $S$. Let $\mathcal{U}$ denote the set of all geodesics that are nonzero in $S$. For $n \geq 2$, let $\mathcal{U}_{n}$ denote the subset of geodesics whose word trees have at most $n$ leaf vertices. Since a word tree has at most two more vertices than the number of idempotents in the Schein canonical form, we have $\mathcal{U}=\mathcal{U}_{M+2}$, by Scholium 2.2. We prove that $\mathcal{U}_{n}$ has bounded Shirshov height by induction, for $n=2, \ldots, M+2$. By Theorem 4.2, there exists a set $X$ of reduced words such that $T$ has bounded Shirshov height $k$ with respect to $X$ and the alphabet $B$. Observe first that

$$
\mathcal{U}_{2}=\left\{g^{-1} g w h h^{-1} \mid g, h, g w h \in T\right\}
$$

so that the Shirshov height of $\mathcal{U}_{2}$ is bounded by $3 k$, which starts an induction. Note the height is Shirshov because each reduced word in the factorisation is expressed 
as a product of powers of elements of $X$ using usual concatenation of words in the free semigroup $F_{B}$. This same observation will hold in the inductive step below (without comment). Suppose $n>2$ and, as inductive hypothesis, that $\mathcal{U}_{n-1}$ has Shirshov height bounded by $k^{\prime}$ with respect to $X$. We claim that $\mathcal{U}_{n}$ has Shirshov height bounded by

$$
K=(M+1) k^{\prime}+2 k
$$

where $M$ is the constant in Scholium 2.2. Consider a geodesic word of the form

$$
w \quad \underline{\overline{0}} u_{0} e_{1} u_{1} e_{2} \ldots e_{m} u_{m}
$$

that is not in $\mathcal{U}_{2}$. If $u_{0}$ is nonempty then $T\left(e_{1} u_{1} e_{2} \ldots e_{m} u_{m}\right)$ has one less leaf vertex than $T(w)$, so by the inductive hypothesis, $w$ has height bounded by

Suppose then that

$$
k+k^{\prime} \leq K
$$

$$
w \quad \overline{\mathrm{o}} \quad e_{1} u_{1} e_{2} \ldots e_{m} u_{m}
$$

where $e_{1}$ is the (nonempty) label of a tree emanating from the initial vertex of $T(w)$. If $T\left(e_{1}\right)$ is a chain then $u_{1}$ and $e_{2}$ are nonempty (since $w \notin \mathcal{U}_{2}$ ), so $e_{1} \overline{\mathrm{o}} g^{-1} g$ for some reduced word $g$ such that $g u_{1}$ is reduced, and $T\left(e_{2} u_{2} \ldots e_{m} u_{m}\right)$ has one fewer leaf vertex than $T(w)$, so, by the inductive hypothesis, $w$ has height bounded by

$$
2 k+k^{\prime} \leq K
$$

Suppose then that $T\left(e_{1}\right)$ is not a chain. There are two subcases. Suppose first that the degree of the initial vertex of the word tree $T(w)$ is $\delta>2$, so that $e_{1}$ 의 $f_{1} \ldots f_{\delta-1}$ where $f_{1}, \ldots, f_{\delta-1}$ label idempotents corresponding to branches emanating from the initial vertex. But $T\left(f_{1}\right), \ldots, T\left(f_{\delta-1}\right)$ and $T\left(u_{1} e_{2} \ldots e_{m} u_{m}\right)$ have fewer than $n$ leaf vertices, and $\delta-1 \leq M$ by Scholium 2.2. Hence $w$ has height bounded by

$$
(\delta-1) k^{\prime}+k^{\prime} \leq(M+1) k^{\prime} \leq K
$$

Finally, suppose the degree of the initial vertex is 2 . Then

$$
e_{1} \quad \overline{\mathrm{o}} z g_{1} \ldots g_{\ell-1} z^{-1}
$$

for some reduced word $z$ labelling a path from the initial vertex to a vertex of degree $\ell$ say, from which there are $\ell-1$ branches corresponding to idempotents labelled by $g_{1}, \ldots, g_{\ell-1}$. Now $T\left(g_{1}\right), \ldots, T\left(g_{\ell-1}\right)$ and $T\left(u_{1} e_{2} \ldots e_{m} u_{m}\right)$ have fewer than $n$ leaf vertices, so $w \quad \underline{\overline{0}} z g_{1} \ldots g_{\ell-1} z^{-1} u_{1} e_{2} \ldots e_{m} u_{m}$ has height bounded by

$$
k+(\ell-1) k^{\prime}+k+k^{\prime} \leq(M+1) k^{\prime}+2 k=K,
$$

and the proof of the theorem is complete. 
We remark that the equivalence of (a) and (d) is also proved, by different means, in [9, Theorem 4.3.6].

As an application of the equivalence of (a) and (b) of the previous theorem, we prove Theorem 4.4 below, which may be regarded as an inverse semigroup analogue of the following classical result: consider a finitely presented semigroup $\Pi$ with zero such that all relations have zero on the right hand side (that is, $\Pi$ is a finitely presented Rees quotient of a free semigroup). Then $\Pi$ has polynomial growth if and only if each nonzero element of $\Pi$ that is not nilpotent can be represented by a word whose primitive root labels some loop of the Ufnarovsky graph of the presentation. This follows from [6] where the syntactic structure of the Jacobson radical was described (also see this description in [24, Section 7.6, Theorem 3]) and the trivial fact that $\Pi$ embeds in an associative monomial algebra with the same set of generators and defining relations (see also [13, Chapter 24]).

TheOREm 4.4. Let $S$ belong to the class $\mathfrak{M}_{F I}$. The following conditions are equivalent:

(a) $S$ has polynomial growth.

(b) If $u$ is a word over $B$ such that $u$ is not nilpotent in $S$, then either $u$ is idempotent, or $u$ 으 $x^{-1} x w y y^{-1}$ where $w$ 으 $\bar{u}$ is cyclically reduced, $x$ is a suffix of $w, y$ is a prefix of $w$ and $w \overline{\overline{ }} z^{\alpha}(\alpha \geq 1)$ for some primitive word $z$ that labels a loop of the Ufnarovsky graph.

Proof. As before, the equivalence of the conditions is immediate if $\bar{d}$ is zero or does not exist, so we may suppose $\bar{d} \geq 1$.

Suppose first that (a) holds and $u$ is a word over $B$ that is neither idempotent nor nilpotent in $S$. By replacing $u$ by a sufficiently high power, if necessary, we may suppose $\ell(w)>\bar{d}$ where $w \underline{\overline{0}} \bar{u}$. By [21, Lemma 5.2], $w$ is a cyclically reduced word such that $u$ lies in the closure (in the sense of Schein [17], see also [8] or [14]) of the inverse subsemigroup of $F I_{A}$ generated by $w$. Replacing $w$ by a sufficiently high power, if necessary, we may suppose that $u \underline{\overline{ }} x^{-1} x w y y^{-1}$ where $x$ is a suffix and $y$ is a prefix of $w$. But there is no vertex of $\Gamma_{S}$ contained in different cycles, since $S$ has polynomial growth, so an equation of the form (3) holds:

$$
w \text { 의 } v P_{1}\left(x_{1} y_{1}\right)^{\alpha_{1}} x_{1} P_{2}\left(x_{2} y_{2}\right)^{\alpha_{2}} x_{2} \ldots P_{m}\left(x_{m} y_{m}\right)^{\alpha_{m}} x_{m} P_{m+1} \text {, }
$$

where $c_{j} \overline{\mathrm{o}} \quad x_{j} y_{j}$ are distinct words labelling loops of $\Gamma_{S}$ for $j=1, \ldots, m$. By Lemma 3.1, each $c_{j}$ is primitive. But $w$ is not nilpotent and no vertex of $\Gamma_{S}$ is contained in different cycles, so it follows quickly that $m=1$ and

$$
w \text { 의 } v P_{1} c_{1}^{\alpha_{1}} \text { 으 } z^{\alpha}
$$

for some positive integer $\alpha>\alpha_{1}$, where $z$ 으 $c_{1}$, completing the proof that (b) holds. 
Suppose conversely that (b) holds. We first prove that no vertex of $\Gamma_{S}$ lies in distinct cycles. Suppose to the contrary that $\Gamma_{S}$ has a vertex $v$ in two distinct cycles, which we may assume are labelled by words $u x$ and $w x$ respectively where $x$ is the last letter of $v$ and $u$ and $w$ begin with different letters. For any positive integers $\beta$ and $\gamma$, consider the word

$$
D_{\beta, \gamma} \quad \underline{\overline{0}}(u x)^{\beta}(w x)^{\gamma} .
$$

Then $D_{\beta, \gamma}$ labels a path in $\Gamma_{S}$, so certainly $D_{\beta, \gamma}$ is not nilpotent in $S$. But we may take one of $\beta$ or $\gamma$ sufficiently large so that $D_{\beta, \gamma}$ is not a power of a word of length less than or equal to the maximum length of all reduced words labelling loops of $\Gamma_{S}$. This violates condition (b). Hence no vertex of $\Gamma_{S}$ lies in distinct cycles. By Theorem 4.2, the set of all reduced words that are nonzero in $S$ has bounded height. But also by (b), every reduced but not cyclically reduced nonzero word in $S$ must be nilpotent. Hence condition (b) of Theorem 4.3 holds, so that (a) holds, completing the proof of the theorem.

\section{ACKnowledgement}

The authors gratefully acknowledge the support of the Australian Research Council and the School of Mathematics and Statistics at the University of Sydney, which made possible this collaborative work. The work of the first author was also partially supported by the PSC-CUNY Research Award Program.

\section{REFERENCES}

[1] H. Bass, "The degree of polynomial growth of finitely generated nilpotent groups", Proc. London Math. Soc. 25 (1972), 603-614.

[2] G. Baumslag and D. Solitar, "Some two-generator one-relator non-Hopfian groups", Bull. Amer. Math. Soc. 68 (1962), 199-201.

[3] A.H. Clifford and G.B. Preston, The algebraic theory of semigroups, Math. Surveys No. 7, Amer. Math. Soc., Providence, Vol. I (1961).

[4] C. Choffrut, "Conjugacy in free inverse monoids", Internal. J. Algebra Comput. 3 (1993), 169-188.

[5] D. Easdown and L.M. Shneerson, "Principal Rees quotients of free inverse semigroups", Glasgow Math. J. 45 (2003), 263-267.

[6] T. Gateva-Ivanova and V.N. Latyshev, "On recognisable properties of associative algebras", J. Symb. Comput. 6 (2/3) (1988), 371-388.

[7] P.M. Higgins, Techniques of semigroup theory, Oxford University Press, 1992.

[8] J.M. Howie, An introduction to semigroup theory, Academic Press, 1976. 
[9] J. Lau, Growth of a class of inverse semigroups, $\mathrm{PhD}$ thesis, University of Sydney, 1997.

[10] J. Lau, "Rational growth of a class of inverse semigroups", J. Algebra 204 (1998), 406-425.

[11] J. Lau, "Degree of growth of some inverse semigroups", J. Algebra 204 (1998), 426-439.

[12] W.D. Munn, "Free inverse semigroups", Proc. Lond. Math. Soc. (3) 29 (1974), 385-404.

[13] J. Okninski, Semigroup algebras, Marcel Dekker, 1980.

[14] M. Petrich, Inverse semigroups, Wiley \& Sons, 1984.

[15] O. Poliakova and B.M. Schein, "A new construction for free inverse semigroups", J. Algebra 288 (2005), 20-58.

[16] L.H. Rowen, Polynomial identities in ring theory, Academic Press, 1980.

[17] B.M. Schein, "Representations of generalised groups", Izv. Vyss. Ucebn. Zav. Matem. 3 (28) (1962), 164-176 (in Russian).

[18] A.I. Shirshov, "On rings with identity relations", Mat. Sb. 43 (1957), 277-283 (in Russian).

[19] L.M. Shneerson, "Identities and a bounded height condition for semigroups", Internat. J. Algebra Comput. 13 (2003), 565-583.

[20] L.M. Shneerson, "Remarks on the growth of inverse semigroups", Contemporary Mathematics 360 (2004), 151-165.

[21] L.M. Shneerson and D. Easdown, "Growth and existence of identities in a class of finitely presented inverse semigroups with zero", Internat. J. Algebra Comput. 6 (1996), 105-121.

[22] A.M. Slinko, I.P. Shestakov, A.I. Shirshov and K.A. Zhevlakov, Rings that are nearly associative, Moscow, 1978.

[23] V.A. Ufnarovsky, "Growth criterion for graphs and algebras given by words", Mat. Zam. 31, No. 3 (1982), 465-472 (in Russian).

[24] V.A. Ufnarovsky, "Combinatorial and asymptotic methods in algebra", Algebra VI, Encyclopaedia Math. Sci., Springer 57 (1994), 1-196.

[25] J. Wolf, "Growth of finitely generated solvable groups and curvature of Riemannian manifolds", J. Differential Geom. 2 (1968), 421-446.

Department of Mathematics and Statistics

Hunter College, The City University of New York 695 Park Avenue

New York, NY 10065, USA
School of Mathematics and Statistics

University of Sydney

Sydney, NSW 2006

Australia 